\title{
COMUNICAÇÃO
}

\section{SERUM BILE ACIDS IN SCHISTOSOMOTIC HEPATIC FIBROSIS}

\section{Edison R. Parise}

Serum bile acid determination has been claimed as a sensitive liver function test in chronic hepatic disease and several papers have been published assessing serum bile acids (more specifically cholilglycine) in hepatic schistosomiasis, suggesting that this determination can be of value in the study of such patients ${ }^{5}$.

Zwingerberger et al $^{6}$ found a distinctive values for hepatointestinal (HI) and hepatoplenic (HS) patients, but could not found differences between compensated and decompensated HS patients with a very similar values for both groups. According to these authors, the increased cholilglycine concentration in the serum of HS patients, would be related to the modifications in the sinusoidal endothelium and the bypass of the liver by the porto-caval shunting. These findings would be in agreement with experimental studies, where a temporal association between the development of hepatic fibrosis in murine hepatic schistosomiasis and increasing cholilglycine concentration in the peripheral blood is found ${ }^{1}$.

Such proposition is not entirely convincing, since it is believed that sinusoidal morphological fibrosis is normally found in patients with the decompensated form of HS schistosomiasis ${ }^{2}{ }^{3}$. Furthermore if the spillover of bile acids to the systemic circulation because of porto-caval shunting resulting from portal hypertension, would increase the cholilglycine levels in the serum, the overnight fasting can recycle the bile acids several times through the liver circulation, and the fasten levels of cholylglycine could be normalized, depending on the hepatic function. This theoretical hypothesis can be supported by the data obtained in hepatic cirrhosis where several patients with established hepatic cirrhosis present normal serum bile acids levels.

In our previously published work ${ }^{5}$ the mean values for fasting serum cholilglycine was more

\footnotetext{
Escola Paulista de Medicina, São Paulo, SP.

Endereço para correspondência: Dr. Edison R. Parise. Disc. de Gastroenterologia/EPM. CP: 20207, 04034-970 Sāo Paulo, SP.

Recebido para publicação em 25/07/92.
}

elevated in compensated HS group than in $\mathrm{HI}$ schistosomiasis but it did not reach the significance level. The more striking finding was the significant increment in the bile acid concentration in the HS decompensated group when compared with all the other two groups. More interesting, the serum cholylglycine determination two hours after a standart meal, showed significant differences between the HI and the two forms of presentation of $\mathrm{HS}$ schistosomiasis. Such postprandial determination should be more able to disclose modifications due to the spillover of such compounds, since no time would be available for SBA recycling (Figure 1).

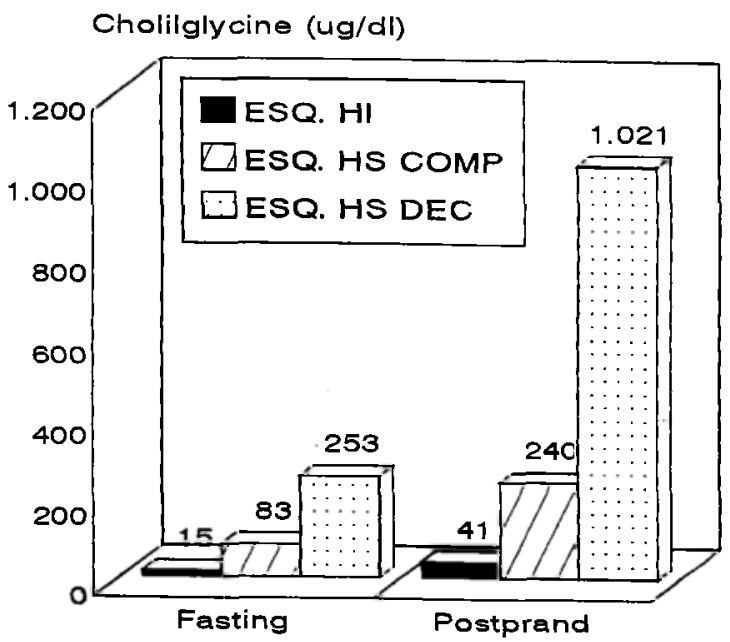

Figure I - Serum bile acids in schistosomiasis.

The correlation of the different biochemical or enzymatic parameters assessed in all the schistosomotic patients, with the 30 minutes bromsulphalein (BSP) retention test, taken as referential "golden standard", showed that cholilglycine determination was the single test that best correlated with the BSP retention in those schistosomotic patients.

Some differences between those published papers could be due the fact that Zwingerberger et al seemed to include intestinal hemorrhage as a criteria of hepatic decompensation and did not assessed other biochemical parameters of hepatocellular function. 